\title{
Tips for Dealing with Common Beginner's Mistakes Made during Endoscopic Ultrasound-Guided Gallbladder Drainage
}

\author{
Min Jae Yang Jae Chul Hwang Byung Moo Yoo Jin Hong Kim \\ Department of Gastroenterology, Ajou University School of Medicine, Suwon, South Korea
}

\section{Keywords}

Endoscopic ultrasound - Endoscopic ultrasound-guided gallbladder drainage - Acute cholecystitis - Technical failure . Stent misplacement

\begin{abstract}
Endoscopic ultrasound-guided gallbladder drainage (EUSGBD) has been performed as an alternative to percutaneous drainage in surgically high-risk patients. Technical failures of EUS-GBD made by beginners are often attributed to failure of over-the-wire insertion of a fistula-dilating device or stent delivery system into the gallbladder, or stent misplacement in the final technical step. We herein report technical tips to prevent the failure of fistula dilation and provide tricks to avoid inward or outward stent misplacement.
\end{abstract}

(c) 2020 S. Karger AG, Basel

\section{Introduction}

Endoscopic ultrasound-guided gallbladder drainage (EUS-GBD) has been accepted as an alternative option to percutaneous cholecystostomy in patients at high risk for cholecystectomy [1-4]. Coiling of a guidewire within the gallbladder (GB), fistula dilation, and eventual proper positioning of a stent are essential prerequisites for successful EUS-GBD [5]. Technical failures of EUS-GBD made by beginners are often attributed to failure of over-thewire insertion of a fistula-dilating device or stent delivery system into the GB $[1,6,7]$, or stent misplacement in the final technical step $[3,8,9]$. Although most previous trials have reported technical failures of EUS-GBD with numerical data, few studies have reported details about technical failure and countermeasures for dealing with difficult situations in which technical failure is imminent. In this case series, we report technical tips to prevent the tangential advancement of a fistula-dilating device toward the GB wall, which can lead to an undesirable failure of fistula dilation. We also provide tricks to avoid immediate inward or outward stent misplacement.

\section{Case Presentations}

Case 1

A 90-year-old woman presented with progressive worsening of fever and epigastric pain caused by acute calculous cholecystitis. EUS-GBD was discussed with the patient because she was a surgically high-risk candidate for cholecystectomy. Her GB was punctured from the duodenal wall with a 19-gauge needle (EUSN-19-T; karger@karger.com

www.karger.com/ddi

Karger $\stackrel{2}{=}$ (c) 2020 S. Karger AG, Base
Byung Moo Yoo, MD, PhD or Jin Hong Kim, MD, PhD

Department of Gastroenterology, Ajou University School of Medicine 164 Worldcup-ro, Yeongtong-gu

Suwon-si, Gyeonggi-do 16499 (South Korea)

ybm6403@gmail.com or jinhkim@ajou.ac.kr 


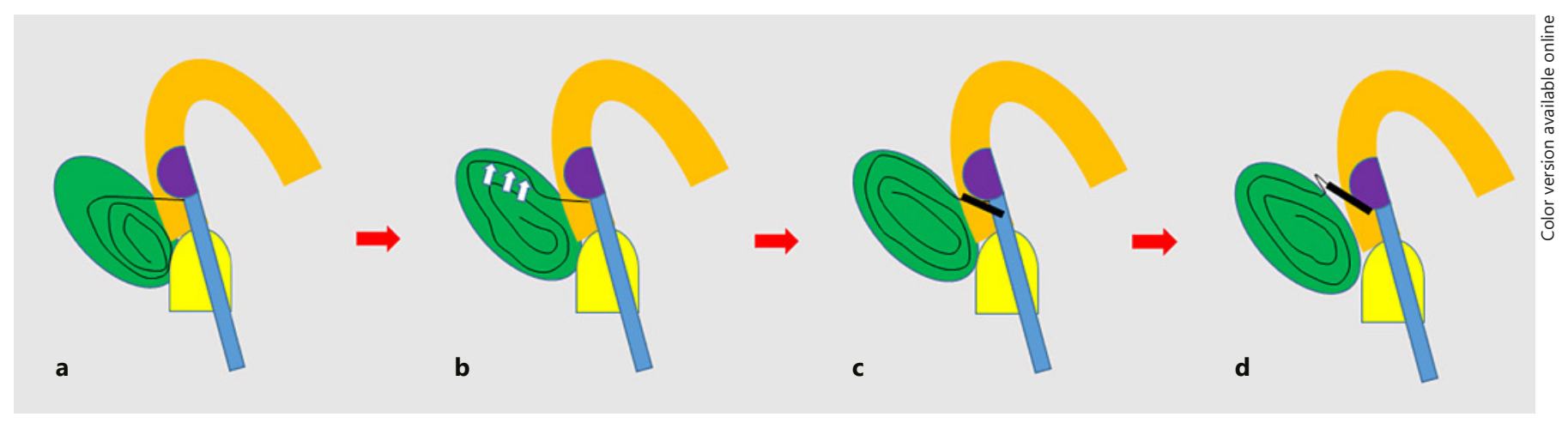

Fig. 1. Tangential advancement of a fistula-dilating device to the gallbladder wall, leading to an undesirable failure of fistula dilation.

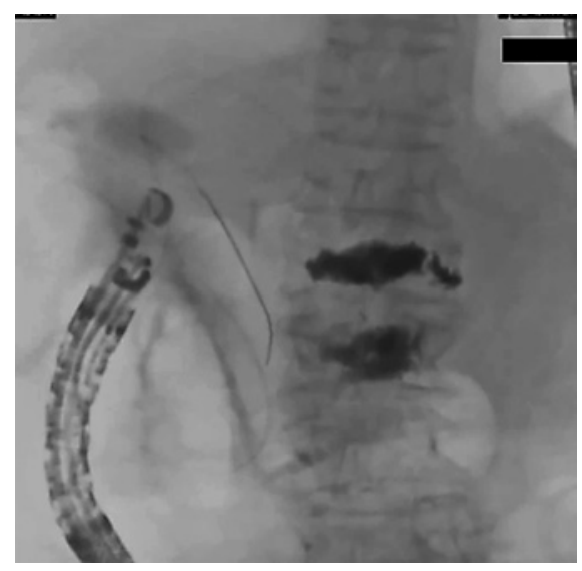

Video 1. Failure of fistula dilation (case 1).

Cool Endoscopy) and a 0.025-inch guidewire (VisiGlide; Olympus). Although the guidewire was left sufficiently indwelling in the GB (Fig. 1a), forced guidewire coiling continued against resistance, leading to larger loop formation in the guidewire within the GB. As a result, the direction of the guidewire just entering into the GB lumen changed from perpendicular to parallel relative to the GB wall, compared with the direction at the time of needle puncture (Fig. 1b). Therefore, the sequential over-the-wire cautery-dilating device (Microtome; Boston Scientific) was advanced with tangential direction toward the GB wall (Fig. 1c), and the device eventually failed to penetrated the GB wall; instead, it repetitively slid along its surface (Fig. 1d). We used a metal clip to seal the defect of the duodenal entry site (Video 1). The patient was then referred to interventional radiology for percutaneous cholecystostomy after successful endoscopic closure. The post-procedural course was fortunately uneventful.

Case 2

A 92-year-old woman was referred to our hospital for fever and right upper quadrant pain caused by acute calculous cholecystitis. Considering the high surgical risk due to her age, EUS-GBD was performed. An 8-Fr stent delivery sheath comprising a tubular covered metal stent $(10 \mathrm{~mm} \times 7 \mathrm{~cm}$ BONA-AL stent; Standard Sci-
Tech Inc.) was introduced into the GB. After the GB part of the stent was successfully deployed in the GB lumen, the stent delivery sheath was slowly pulled back to deploy the remaining duodenal portion of the stent. At that, the GB side of the stent inadvertently slid out of the GB lumen with the tip of the stent becoming lodged in the GB wall, and the duodenal side of the stent was displaced into the gastric antrum across the pyloric ring (Fig. 2a, b). Fortunately, the guidewire was retained inside the GB.

For rescue, the edge of the dislocated stent was grasped using rat-tooth forceps alongside the guidewire in the gastric antrum (Fig. 2c, d). Thereafter, the stent was pulled out over the guidewire through the working channel of the echoendoscope while the guidewire was kept coiled in the GB (Fig. 2e). Even though guidewire shearing occurred during stent withdrawal, a rescue lumenapposing metal stent $(10 \mathrm{~mm} \times 2 \mathrm{~cm}$ SPAXUS; Taewoong) was advanced over the guidewire and appropriately placed, successfully bridging the GB and duodenal lumen (Fig. 2f; Video 2).

Case 3

A 94-year-old man with distal cholangiocarcinoma underwent endoscopic retrograde biliary drainage with a self-expandable metal stent for distal biliary obstruction. He was readmitted for fever and right upper quadrant pain caused by acute cholecystitis. We decided to perform EUS-GBD because of the high surgical risk due to his age. The fistula was dilated using a triple-lumen needle knife (Microtome; Boston Scientific). While introducing the delivery sheath of the lumen-apposing metal stent $(10 \mathrm{~mm} \times 2 \mathrm{~cm}$ SPAXUS; Taewoong $)$ into the GB across the fistula, the EUS view plane where the delivery sheath was observed via EUS imaging could not be maintained because of tissue resistance and unstable scope positioning; thus, the GB flange of the stent was deployed in the GB neck under fluoroscopic guidance. Thereafter, the deployed stent portion on the GB side was clamped in the confined space of the GB neck, and the stent delivery sheath could not be pulled back sufficiently. The unreleased enteral portion of the stent was deployed inadvertently while repetitively pulling the delivery sheath, with the enteral flange stuck in the duodenal wall (Fig. 3a, b). For rescue, a covered metal stent $(10 \mathrm{~mm} \times 7 \mathrm{~cm}$ BONA-AL stent; Standard Sci-Tech Inc.) was successfully inserted over the retained guidewire across the collapsed enteral side lumen of the misplaced lumen-apposing stent, albeit with considerable resistance when advancing the stent delivery sheath (Fig. 3c, d; Video 3). 

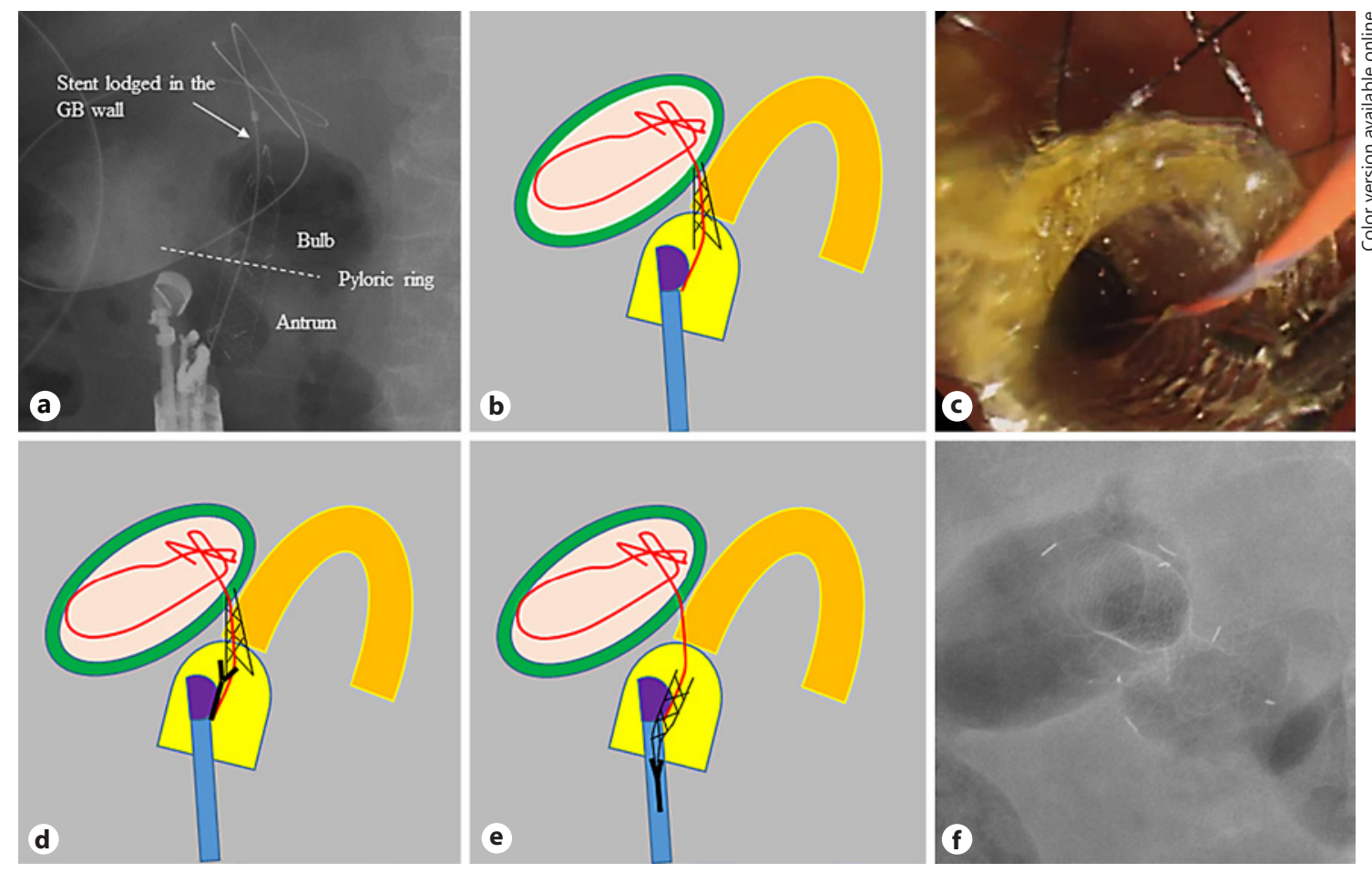

Fig. 2. Salvage technique for immediate inward misplacement of the covered metal stent during endoscopic ultrasonography-guided gallbladder drainage. GB, gallbladder.

\section{Discussion}

Sequential insertion of a fistula-dilating device and stent delivery system into the GB is a crucial technical step for successful EUS-GBD. However, those devices may not penetrate the GB wall but slide along its surface, even though the guidewire is coiled within the GB. That phenomenon can occur when the direction of the device is tangential to the GB wall. At the time of needle puncture, the direction of the guidewire just entering the GB is usually perpendicular to the GB wall, but excessive coiling of the indwelling guidewire within the GB can cause larger loop formation in the coiled guidewire, leading to a change of axis of the guidewire entering the GB in a direction parallel or tangential to the GB wall. This change in axis of the guidewire can directly lead to failure of fistula dilation, albeit successful guidewire coiling within the GB. Therefore, the operator should concentrate on maintaining the position and direction of the guidewire portion entering into the GB lumen during the guidewire coiling process, similar to the initial puncture step.

Inward stent misplacement can occur during stent delivery sheath withdrawal within the echoendoscope for final release of the stent. The stent can slide back into the

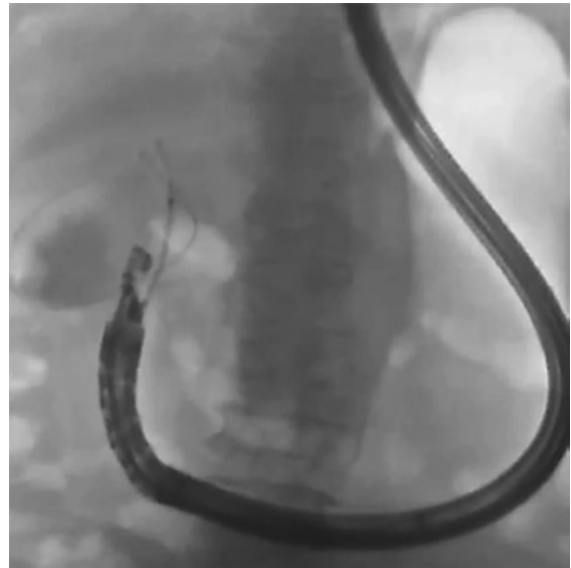

Video 2. Inward stent misplacement (case 2).

duodenum because the transmural fistula that is created does not have a true fibrotic stricture and may not act as tight clamping points for anchoring the metal stent. Unlike cases with immediate outward misplacement wherein an additional long stent can be instantly inserted inside the misplaced stent, stent removal is inevitable in inward stent misplacement cases if the deployed portion of the 
Fig. 3. Salvage technique for immediate outward misplacement of the lumen-apposing metal stent during endoscopic ultrasound-guided gallbladder drainage.
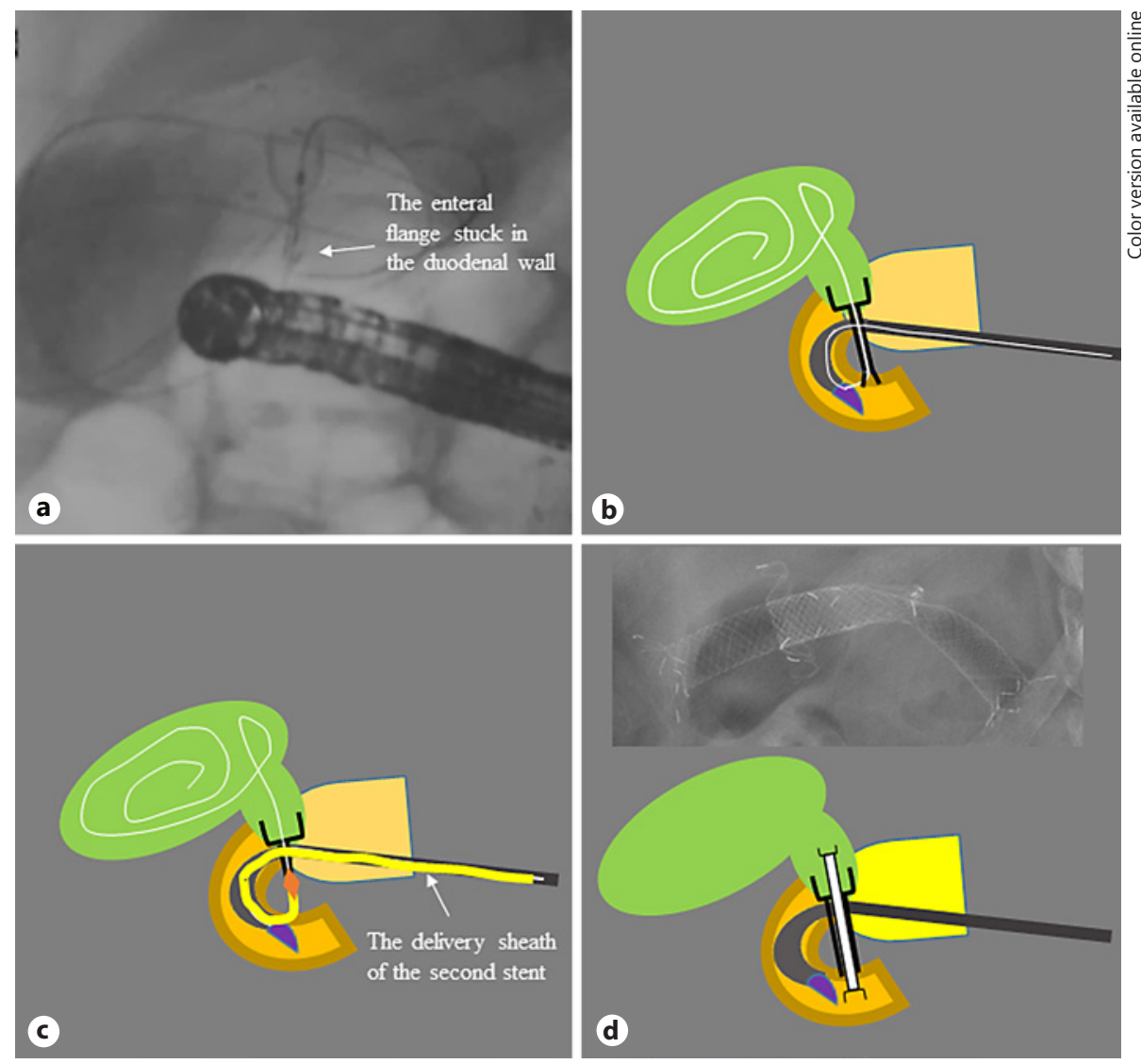

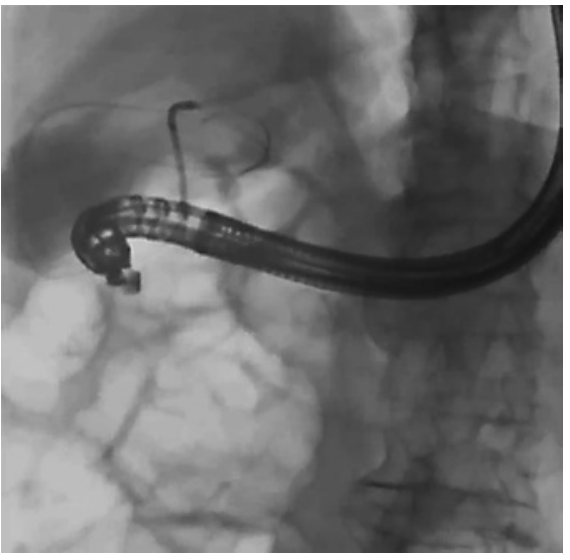

Video 3. Outward stent misplacement (case 3).

stent is not re-sheathed by repositioning the stent deployment hub to the original position [10]. The displaced stent should be retrieved over the guidewire through the working channel of the echoendoscope while maintaining the guidewire's and echoendoscope's positions.
Outward stent misplacement into the abdominal cavity is a troublesome adverse event during EUS-guided biliary drainage [11]. During EUS-GBD, the enteral flange of the lumen-apposing metal stent can be immediately deployed into the abdominal cavity when the stent portion that is already deployed in the GB is clamped and fixed in the narrow space of the GB neck; however, the position of the echoendoscope is relatively unstable and unfixed in the duodenal lumen. In this situation, the actual positions of the stent and delivery sheath remain unchanged, even after the sheath is pulled back; instead, the scope paradoxically moves forward. This optical illusion can cause the operator to mistakenly think that the delivery sheath is pulled out sufficiently through the working channel of the scope for intra-working channel stent release. This may lead to misplacement of the enteral flange of the lumen-apposing metal stent into the duodenal wall or intraabdominal cavity during the EUSGBD procedure. If the tip of the stent is visible in the duodenal wall, pulling the stent with grasping forceps can be attempted to open the enteral end in the duodenum. However, if it fails, an additional longer stent 
should be deployed within the first stent over the retained guidewire across the collapsed enteral side of the misplaced stent.

\section{Acknowledgement}

We thank Sang Hun Lee, RN, Kwon Soon Kim, RN, Sang Heon Lee, $\mathrm{RN}$, and all the nursing staff for assistance with the procedures.

\section{Statement of Ethics}

All patients provided written informed consent before undergoing EUS-GBD. This study was conducted according to the ethical principles of the Declaration of Helsinki, and approved by our institute's Committee on Human Research.

\section{Disclosure Statement}

The authors have no conflicts of interest or financial ties to disclose.

\section{Author Contributions}

M.J. Yang: planned the study, wrote the initial draft, and revised the manuscript. B.M. Yoo: wrote the initial draft and critically revised the manuscript. J.H. Kim: critically revised and finalized the manuscript. J.C. Hwang: analyzed data.

\section{References}

1 Jang JW, Lee SS, Song TJ, Hyun YS, Park DY, Seo DW, et al. Endoscopic ultrasound-guided transmural and percutaneous transhepatic gallbladder drainage are comparable for acute cholecystitis. Gastroenterology. 2012 Apr; 142(4):805-11.

2 Choi JH, Lee SS, Choi JH, Park DH, Seo DW, Lee SK, et al. Long-term outcomes after endoscopic ultrasonography-guided gallbladder drainage for acute cholecystitis. Endoscopy. 2014 Aug;46(8):656-61.

3 Teoh AY, Serna C, Penas I, Chong CC, PerezMiranda M, Ng EK, et al. Endoscopic ultrasound-guided gallbladder drainage reduces adverse events compared with percutaneous cholecystostomy in patients who are unfit for cholecystectomy. Endoscopy. 2017 Feb;49(2): $130-8$.

4 Tyberg A, Saumoy M, Sequeiros EV, Giovannini M, Artifon E, Teoh A, et al. EUS-guided Versus Percutaneous Gallbladder Drainage:
Isn't It Time to Convert? J Clin Gastroenterol. 2018 Jan;52(1):79-84.

5 Choi JH, Lee SS. Endoscopic ultrasonography-guided gallbladder drainage for acute cholecystitis: from evidence to practice. Dig Endosc. 2015 Jan;27(1):1-7.

6 Choi JH, Kim HW, Lee JC, Paik KH, Seong NJ, Yoon CJ, et al. Percutaneous transhepatic versus EUS-guided gallbladder drainage for malignant cystic duct obstruction. Gastrointest Endosc. 2017 Feb;85(2):357-64.

7 Minaga K, Yamashita Y, Ogura T, Takenaka M, Shimokawa Y, Hisa T, et al. Clinical efficacy and safety of endoscopic ultrasound-guided gallbladder drainage replacement of percutaneous drainage: A multicenter retrospective study. Dig Endosc. 2019 Mar;31(2):180-7.

8 Law R, Grimm IS, Stavas JM, Baron TH. Conversion of Percutaneous Cholecystostomy to Internal Transmural Gallbladder Drainage Using an Endoscopic Ultrasound-Guided,
Lumen-Apposing Metal Stent. Clin Gastroenterol Hepatol. 2016 Mar;14(3):476-80.

9 Irani S, Ngamruengphong S, Teoh A, Will U, Nieto J, Abu Dayyeh BK, et al. Similar Efficacies of Endoscopic Ultrasound Gallbladder Drainage With a Lumen-Apposing Metal Stent Versus Percutaneous Transhepatic Gallbladder Drainage for Acute Cholecystitis. Clin Gastroenterol Hepatol. 2017 May;15(5):738-45.

10 Ligresti D, Amata M, Granata A, Cipolletta F, Barresi L, Traina M, et al. Salvage procedure following lumen-apposing metal stent maldeployment during endoscopic ultrasoundguided biliary drainage. Endoscopy. $2018 \mathrm{Jul}$; 50(7):E190-1.

11 Yang MJ, Kim JH, Kim DJ, Hwang JC, Yoo BM. Hepatobiliary and Pancreatic: EUSguided reintervention for extraluminal stent migration after EUS-guided hepaticogastrostomy. J Gastroenterol Hepatol. 2018 Apr; 33(4):772. 